\title{
Guidewire Knot Formation
}

\author{
Hiroaki Saito $^{1}$, Tsuyoshi Suda ${ }^{1}$, and Yoji Nishida ${ }^{1}$ \\ ${ }^{1}$ Kanazawa Municipal Hospital
}

August 28, 2020

\begin{abstract}
There are various complications of PICC, bloodstream infection is well known, but the formation of guidewire knots during PICC insertion is rarely reported. Considering the risk of vascular injury in our case, surgical guidewire removal was performed. Although rare, it is necessary to recognize proper treatment for these complications.
\end{abstract}

\section{Case history/examination:}

An 85-year-old woman was admitted to our hospital for treatment of a liver abscess. To ensure nutritional intake following the patient's loss of appetite, we decided to use a peripherally inserted central catheter (PICC) to facilitate total parenteral nutrition.

The right basilic vein of the forearm was selected and punctured using an ultrasonic guide, and a guidewire was inserted into the vein. At approximately $10 \mathrm{~cm}$, the guidewire encountered strong resistance, and we were unable to remove the guidewire from the patient's body. Radiography indicated that a knot had formed at the tip of the guidewire (Figure 1).

As there was risk of crushing or breaking from forced removal of the guidewire, surgical intervention was performed under local anesthesia for safe removal. Intraoperative findings revealed that the guidewire had penetrated the vein wall and formed a knot outside the wall (Figure 2). No complications occurred after surgery. Although central venous access catheters are known to cause various complications, this is, to our knowledge, the first report describing knot formation of the guidewire during PICC insertion. Previous reports have shown that forced insertion or withdrawal of a knotted guidewire may cause tearing of the veins and that surgical intervention is appropriate in such cases. ${ }^{1,2}$ Although this is a rare complication, it is important for clinicians to know that the guidewire can become knotted and be aware of appropriate treatment methods such as surgical intervention.

\section{Figure Legends:}

Figure 1: Radiograph indicating a knot-like appearance at the tip of the guidewire.

Figure 2: Intraoperative findings showing the guidewire penetrating the vein wall and forming a knot outside the vein.

\section{References}

1. Khan ZH, Tabatabhai SA. 1996. Complication of catheter knotting after right cephalic vein cannulation. Anesth Analg 82:215-216.

2. Kim M, Lee D, Choi D-J. 2012. Guidewire knot formation during angiography through the radial artery. J Clin Exp Cardiolog ;3:4. 


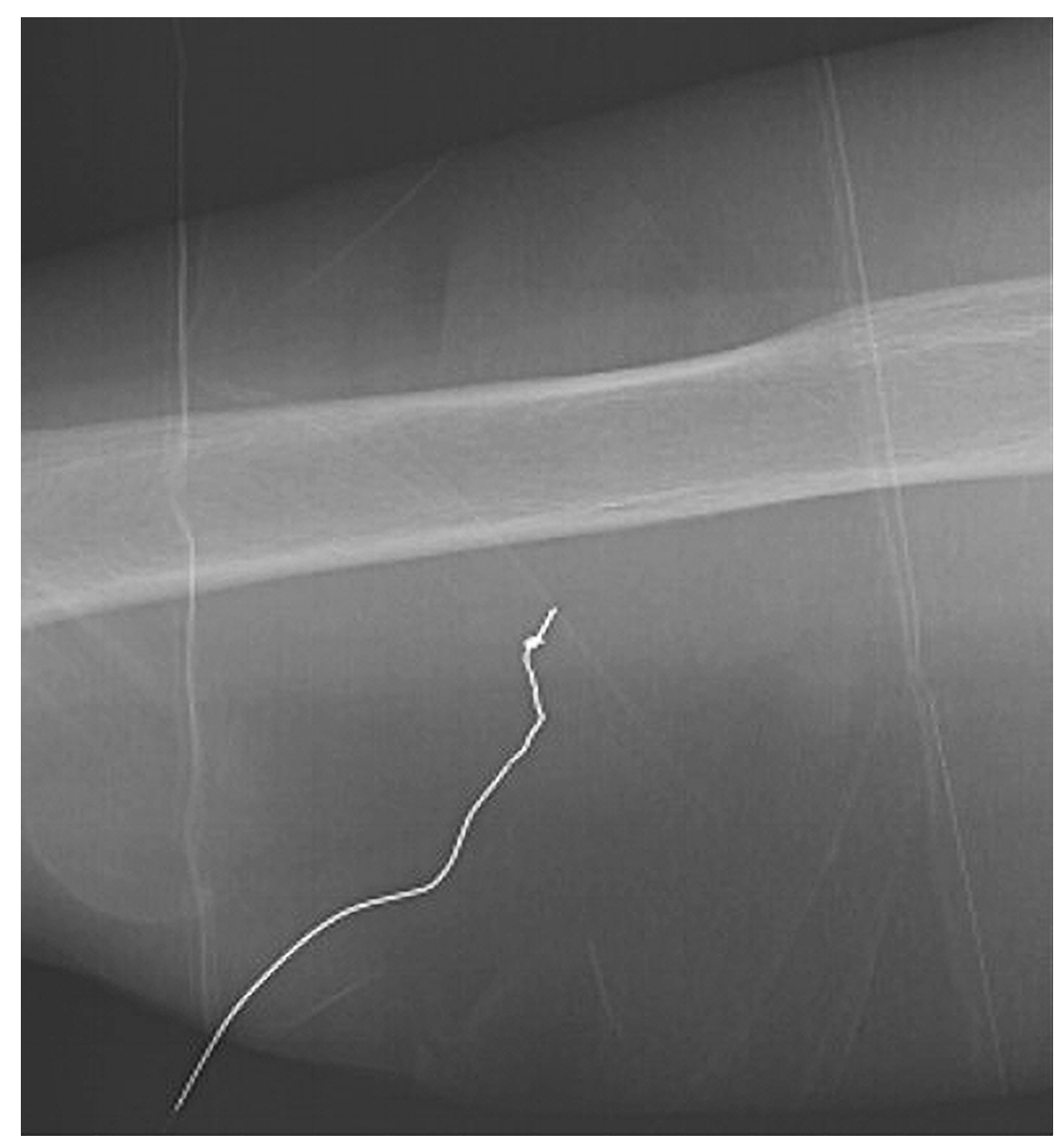




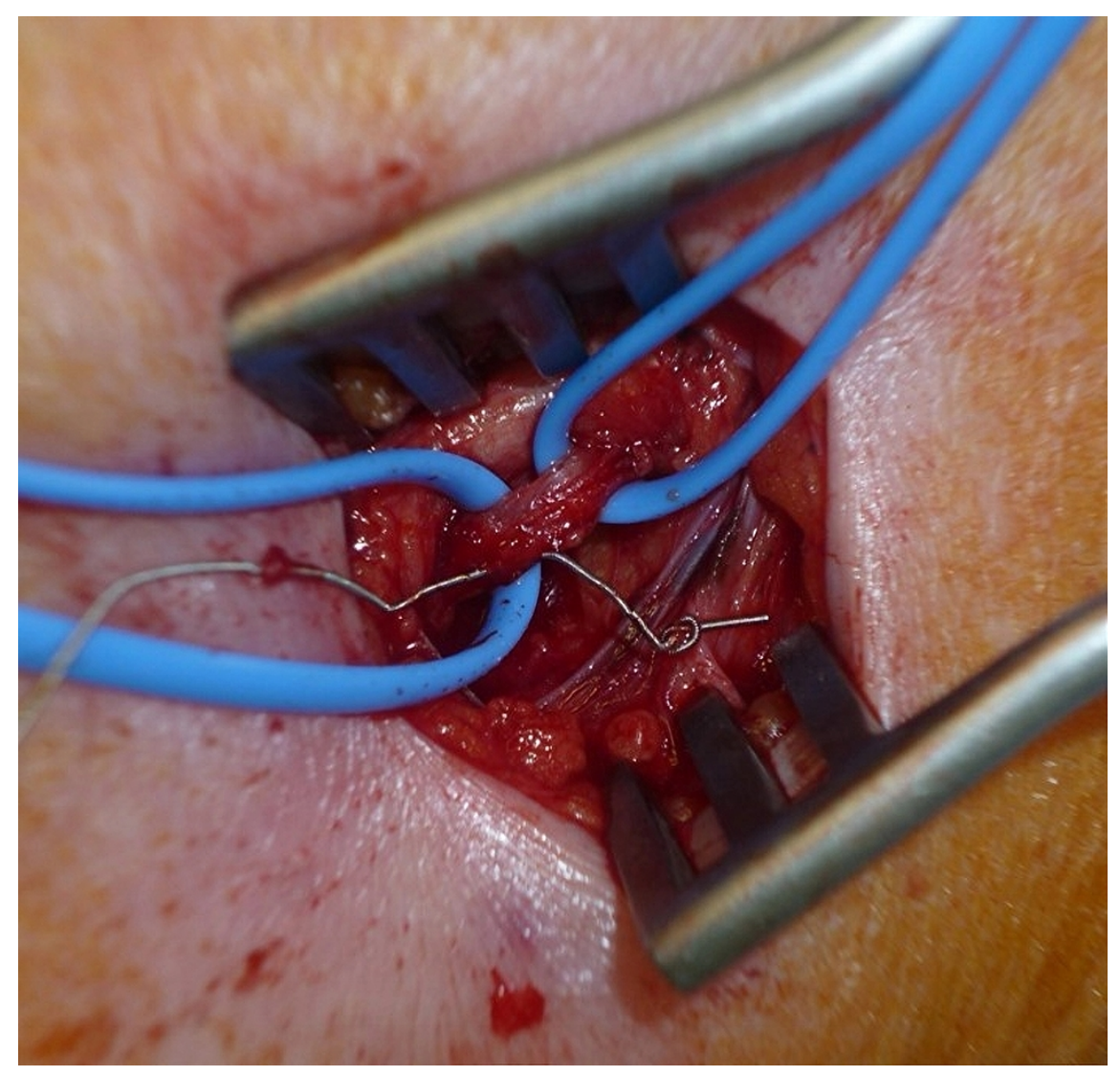

